Pacific Journal of Mathematics

EQUIDISTRIBUTION THEORY IN HIGHER DIMENSIONS 


\title{
EQUIDISTRIBUTION THEORY IN HIGHER DIMENSIONS
}

\section{Chia-Chi Tung}

\begin{abstract}
Let $X, Y$ be complex spaces, and $f: X \rightarrow Y$ a meromorphic map. Assume in $Y$ an admissible family $\mathfrak{A}=\left\{S_{b}\right\}_{b \in N}$ of analytic subsets $S_{b}$ is given. Assume $f$ is almost adapted to $\mathfrak{A}$. The purpose of this paper is to prove that, if $f$ satisfies certain growth conditions, the valence of $S_{b}$ (for almost all $S_{b} \in \mathfrak{U}$ ) grows to infinity at the same rate as the characteristic of $f$. Here $X$ is assumed to carry an exhaustion function which is, e.g., $g$-concave, centrally $g$-convex or $g$-quasiparabolic.
\end{abstract}

The results obtained generalize the Casorati-Weierstrass type theorems of Chern [4] [6], Cowen [7], Griffiths-King [12], Stoll [23] [26], Wu [31, II-III] (see also Griffiths [10]).

Introduction. It is well-known that the classical CasoratiWeierstrass theorem is not true in higher dimensions. In fact, the standard example of Fatou-Bierberbach [2, p. 45] gives a holomorphic imbedding of $\boldsymbol{C}^{2}$ into $\boldsymbol{P}_{2}$ with a nondense image. Chern [4] first showed that a holomorphic map $f: \boldsymbol{C}^{n} \rightarrow \boldsymbol{P}_{n}$ whose characteristic grows sufficiently rapidly assumes almost every point in $\boldsymbol{P}_{n}$. This result was generalized to subvarieties of a general codimension in a complex manifold by Hirshfelder [13] and Stoll [21]-[23]. In Wu [31] certain geometric conditions were given which ensure the Casorati-Weierstrass property. For instance, if $\boldsymbol{C}^{n}$ is given the Fubini-Study metric, then a nondegenerate quasi-conformal holomorphic map $f: \boldsymbol{C}^{n} \rightarrow \boldsymbol{P}_{n}$ assumes almost every point in $\boldsymbol{P}_{n}$. This in fact carries over to a balanced holomorphic map of $\boldsymbol{C}^{m}$ into $\boldsymbol{P}_{n}$ (see [10, p. 54]), whose image intersects almost every $(n-p)$-dimensional linear subspace of $\boldsymbol{P}_{n}$ (where $0<p \leqq \min (m, n))$.

Let $f: X \rightarrow Y$ be a meromorphic map between complex spaces $X, Y$. Assume in $Y$ an admissible family $\mathfrak{A}=\left\{S_{b}\right\}_{b \in N}$ is given. This means $\mathfrak{A}$ is defined by two holomorphic maps $Y \stackrel{h}{\leftarrow} M \stackrel{\pi}{\rightarrow} N$ (where $M$ is a complex space, $N$ a compact complex manifold) such that (i) $\pi$ is open, surjective; (ii) $h$ is proper, locally trivial at every point of $M$; (iii) each $S_{b}$ is the topological image of $\pi^{-1}(b)$ under $h$, and $S_{b}$ contains no branch of $Y$. Then $S_{b}$ is analytic of pure codimension $s$ in $Y$ for all $b$. The main purpose of this paper is to establish the equidistribution property that, for almost every $S_{b} \in \mathfrak{A}$, the valence of $S_{b}$ grows (over suitable sequence of domains) at the same rate as the characteristic of $f$. The admissible family defined here is more 
general than the one given in Stoll [23]. This makes it possible to include, for instance, the Schubert varieties as special cases. Moreover, the Kählerian assumption on the index manifold is no longer required in view of the results of Dektyarev [8] and Stoll [23] (see Theorem 2.5).

The equidistribution theorems are proved in $\S 4$ for different types of spaces. Here only the centrally $g$-convex type will be set out. Let $\varphi$ be a $C^{\infty}$ exhaustion function of $X$. Thus $\varphi: X \rightarrow \boldsymbol{R}$ is a $C^{\infty}$ map such that the sets $X[r]=\{x \in X \mid \varphi(x) \leqq r\}$ are compact for all $r \geqq 0$. Let $L(\varphi)=d d^{c} \varphi$ be the Levi form of $\varphi$. Let $g: \boldsymbol{R}(0, \infty) \rightarrow$ $\boldsymbol{R}$ be an increasing function of class $C^{1}$ with $\left\|e^{-g}\right\|_{1}^{r}=\int_{1}^{r} e^{-g(t)} d t \rightarrow \infty$ as $r \rightarrow \infty$. Then $\varphi$ is said to be centrally $g$-convex $(c . g$-convex) if $X[0]$ has measure zero and if

$L(\varphi) \geqq\left(g^{\prime} \circ \varphi\right) d \varphi \wedge d^{c} \varphi$ off a closed nowhere dense set.

It follows that $L(\varphi) \geqq 0$ on $X$ (Lemma 2.1) and, setting $u=e^{-g}$,

$$
\omega_{u}=(u \circ \varphi)\left[L(\varphi)-\left(g^{\prime} \circ \varphi\right) d \varphi \wedge d^{c} \varphi\right] \geqq 0 \quad \text { on } \quad X-X[0] .
$$

If further

$$
\left(\omega_{u}\right)^{m} \equiv 0 \quad \text { off a compact set }(m=\operatorname{dim} X) \text {, }
$$

then $\varphi$ is called g-semiparabolic. A logarithmic pseudoconvex exhaustion function (in the sense of Stoll [25]) is $g$-convex (with $g=\log$ ). It is not clear to what extent the $g$-convexity generalizes logarithmic pseudoconvexity, except in the trivial case where $g=$ constant (see $\S 4$ for an example).

Assume $\varphi$ is c.g-convex. Define $X(r)=\{x \in X \mid \varphi(x)<r\}, \chi_{p}=$ $L(\varphi)^{p}$. Let $k=\operatorname{dim} N$. Let $\omega_{N, 1}$ be the fundamental form on $N$ of a Hermitian metric normalized so that $\int_{N}\left(\omega_{N, 1}\right)^{k}=1$. The fiber integration operator induced by $h$ is denoted by $h_{*}$. For $\xi \in A_{0}^{p^{\prime}, p^{\prime}}(N)$, $p^{\prime} \geqq k-s$, define $\xi_{Y}=h_{*} \pi^{*} \xi$. Also set $\Omega_{p}=\left(\left(\omega_{N, 1}\right)^{k-s+p}\right)_{Y}, 0 \leqq p \leqq s$. If $r>r^{\prime}>0, p^{\prime}=k-s+p$, and $\xi \geqq 0$, define

$$
\begin{aligned}
D_{f, p}^{u}\left(r, \xi_{Y}\right) & =\int_{X(r)} f^{*} \xi_{Y} \wedge\left(\omega_{u}\right)^{m-p} \\
A_{f, p}^{u}\left(r, \xi_{Y}\right) & =u(r)^{m-p} \int_{X(r)} f^{*} \xi_{Y} \wedge \chi_{m-p} \\
T_{f, p}^{u}\left(r, r^{\prime}, \xi_{Y}\right) & =\int_{r^{\prime}}^{r} A_{f, p}^{u}\left(t, \xi_{Y}\right) u(t) d t .
\end{aligned}
$$

(The existence of the integrals will be established in $\S 4$.)

Theorem. Assume $f: X \rightarrow Y$ is almost adapted to 2 . Assume either $\chi_{q}(q=m-s \geqq 0)$ is semi-positive on an effective open set or 
$\chi_{q} \wedge f^{*} \Omega_{s} \neq \equiv 0$. (1) Assume there exists a positive form $\xi \in A_{0}^{k-1, k-1}(N)$ such that over some $\phi$-admissible sequence $\sigma=\left\{r_{j}\right\}$, one of the following conditions holds:

(a) $A_{f, s-1}^{u}\left(r, \xi_{Y}\right)=o^{\prime}\left(T_{f, s}^{u}\left(r, 1, \Omega_{s}\right)\right)$.

(b) $D_{f, s-1}^{u}\left(r, \xi_{Y}\right)=o^{\prime}\left(\int_{1}^{r} D_{f, s}^{u}\left(t, \Omega_{s}\right) u(t) d t+A_{f, s}^{u}\left(o, \Omega_{s}\right)\|u\|_{1}^{r}\right)$.

Then there is a set $N_{\sigma} \subseteq N$ of measure zero such that for every $b \in N-N_{o}$, the valence

$$
N_{f, s}^{u}\left(r, r_{0}, S_{b}\right)=\int_{r_{0}}^{r} N_{f}\left(X(t), S_{b}, \chi_{q}\right) u(t)^{q+1} d t
$$

grows at the same rate as the characteristic $T_{f, s}^{u}\left(r, r_{0}, \Omega_{s}\right)$ over a subsequence (of $\sigma$ ) $\rightarrow \infty$ depending on $S_{b}$. (2) If $\varphi$ is $g$-semiparabolic and if $s=1$, then taking $\sigma$ to be any $\phi$-admissible sequence, the same conclusion holds. (3) Assume there exists a positive form $\xi \epsilon$ $A_{0}^{k-1, k-1}(N)$ and a positive continuous $\gamma: \boldsymbol{R}\left[a_{0}, \infty\right) \rightarrow \boldsymbol{R}$ with $\|\gamma u\|_{a_{0}}^{r} \rightarrow$ $\infty$ such that for some constants $\alpha>1, B \geqq 0$,

$$
\gamma(r)\left|D_{f, s-1}^{u}\left(r, \xi_{Y}\right)-B\right|^{\alpha}=O\left(D_{f, s}^{u}\left(r, \Omega_{s}\right)\right) \quad(r \longrightarrow \infty) .
$$

Then there is a $\phi$-admissible sequence $\sigma$ for which the conclusion in (1) holds.

A preliminary report of this paper appeared in [29].

1. Adaptation to admissible families. In the following, all complex spaces are reduced, pure dimensional and have a countable basis. A family $\mathfrak{A}=\left\{S_{b}\right\}_{b \in N}$ is said to be admissible in a complex space $Y$ iff:

$\left(\mathrm{A}_{1}\right)$ The index set $N$ is a locally irreducible complex space.

$\left(\mathrm{A}_{2}\right)$ There exists a complex space $M$ and holomorphic maps $\pi: M \rightarrow N, h: M \rightarrow Y$, such that $\pi$ is open, surjective, and $h$ is proper, locally trivial at every point of $M$.

$\left(\mathrm{A}_{3}\right)$ For each $b \in N$ the restriction $h: \pi^{-1}(b) \rightarrow Y$ is injective and $S_{b}=h\left(\pi^{-1}(b)\right)$.

$\left(\mathrm{A}_{4}\right)$ No $S_{b}$ contains a branch of $Y$.

It follows that each $S_{b}$ is an analytic set in $Y$ of pure (constant) codimension $s>0$. If in addition, $h: M \rightarrow Y$ is surjective, then $\mathfrak{A}$ is called strictly admissible (st. adm.).

To give some examples, take integers $p, q, n$ with $0 \leqq p \leqq q \leqq$ $n-1$. Let $V$ be a complex vector space of dimension $n+1$. Let $G_{q}(V)$ be the Grassmann manifold of projective $q$-planes in $\boldsymbol{P}(V)$. If $y \in G_{q}(V)$, the affine $(q+1)$-plane spanned by $y$ is denoted by $E(y)$. Then the flag manifold 


$$
\left.F_{p, q}=\left\{(x, y) \in G_{p}(V) \times G_{q}(y) \mid E(x) \subseteq E(y)\right)\right\}
$$

together with the projections $G_{p}(V) \stackrel{h}{\leftarrow} F_{p, q} \stackrel{\pi}{\rightarrow} G_{q}(V)$ defines a st. adm. family $\mathfrak{A}_{p, q}$ in $G_{p}(V)$ ([22]).

The family $\mathfrak{A}_{0, q}$ belongs to the class of Schubert varieties. Let $A=\left(a_{0}, a_{1}, \cdots, a_{p}\right) \in Z^{p+1}$ with $0 \leqq a_{0} \leqq a_{1} \leqq \cdots \leqq a_{p} \leqq n-p$. The flag manifold of $A$ is the set $F(A)$ of all $v=\left(v_{0}, \cdots, v_{p}\right)$ with $v_{j} \in G_{a_{j}+j}(V)$ such that $E\left(v_{0}\right) \subseteq E\left(v_{1}\right) \subseteq \cdots \subseteq E\left(v_{p}\right)$. For each $v \in \boldsymbol{F}(A)$ the Schubert variety

$$
S_{v}(A)=\bigcap_{j=0}^{p}\left\{x \in G_{p}(V) \mid \operatorname{dim} E(x) \cap E\left(v_{j}\right) \geqq j+1\right\}
$$

has dimension $\sum_{j=0}^{p} a_{j}$. It was shown in Cowen [7] that the Schubert family $\left\{S_{v}(A)\right\}_{v \in F(A)}$ is st. adm. in $G_{p}(V)$. Here the total space $M$ is given by the irreducible complex space

$$
S(A)=\bigcup_{v \in \boldsymbol{F}(A)} S_{v}(A) \times\{v\}
$$

For $b \in G_{n-p-1}(V)$, define

$$
\Sigma_{b}=\left\{x \in G_{p}(V) \mid E(x) \cap E(b) \neq\{0\}\right\}
$$

(see Chern [5, p. 79]). The collection $\mathscr{D}_{p, n}=\left\{\Sigma_{b}\right\}_{b \in G_{n-p-1}(V)}$ is a Schubert family (see below); it is in fact also admissible relative to $G_{n-p-1}(V)$ :

Lemma 1.1. $\mathscr{D}_{p, n}$ is st. adm. in $G_{p}(V)$ of codimension 1.

Proof. Let $A=(n-p-1, n-p, \cdots, n-p) \in \mathbb{Z}^{p+1}$. If $v=$ $\left(v_{0}, \cdots, v_{p}\right) \in \boldsymbol{F}(A)$, the Schubert variety $S_{v}(A)$ has pure codimension 1 in $G_{p}(V)$ and $\Sigma_{v_{0}}=S_{v}(A)$. The unitary group (of a Hermitian metric on $V$ ) acts transitively and biholomorphically on $\boldsymbol{F}(A)$. It follows that the projection $\rho: \boldsymbol{F}(A) \rightarrow G_{n-p-1}(V)$ is open. Hence the triplet $G_{p}(V) \stackrel{h}{\longleftarrow} S(A) \stackrel{\rho \circ \pi}{\longrightarrow} G_{n-p-1}(V)$ is admissible and defines $\mathscr{D}_{p, n}$.

Let $\mathfrak{A}=\left\{S_{b}\right\}_{b \in N}$ be admissible in $Y, s=\operatorname{codim} S_{b}$, and $k=\operatorname{dim} N$. Assume $X$ is a complex space and $f: X \rightarrow Y$ is holomorphic. To obtain the equidistribution property of $f$ rel. to $\mathfrak{A}$, it is necessary to impose a general position requirement on the image set of $f$. Consider the relative fiber product $\left(f^{\prime}, h^{\prime}\right)$ of $(f, h)$ : 


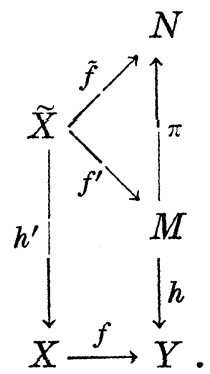

Then $f$ is said to be almost adapted to $\mathfrak{A}$ iff $\widetilde{f}=\pi \circ f^{\prime}$ has strict maximal rank, i.e., the restriction of $\tilde{f}$ to every branch of $\tilde{X}$ has rank $k$. (Observe that if $\operatorname{dim} X<s, f$ is not almost adapted to $\mathfrak{A}_{\text {.) }}$ Take $(a, x) \in N \times X$. The map $f$ is said to be adapted to $a$ at $x$ iff there exist open neighborhoods $U, V$ of $a$, resp. $x$, such that the set $f^{-1}\left(S_{b}\right) \cap V$ either is empty for all $b \in U$ or has pure codimension $s$ in $V$ for all $b \in U$; in the latter case, $f$ is called truly adapted to $a$ at $x$.

Assume now $f: X \rightarrow Y$ is a meromorphic map ([1] [18]). Then $f$ may be thought of as a holomorphic correspondence $[f]: X \rightarrow Y$ (see [20]). Let ' $X \subseteq X \times Y$ be the graph of $[f]$ and $P:{ }^{\prime} X \rightarrow X$, $F:{ }^{\prime} X \rightarrow Y$ be the projections. There is a largest open set $X^{0} \subseteq X$ such that $P: P^{-1}\left(X^{0}\right) \rightarrow X^{0}$ is biholomorphic. Define $f_{0}=F \circ P^{-1}: X^{0} \rightarrow Y$. Then $f_{0}=[f] \mid X^{0}$ is holomorphic. The indeterminacy $I_{f}=X-X^{0}$ is thin analytic in $X$. The map $f$ is said to be: (1) nondegenerate if $F$ has strict maximal rank; (2) almost adapted to $\mathfrak{A}$ if so is $F$.

Define $D=D(\widetilde{F})=\left\{z \in \epsilon^{\prime} \widetilde{X} \mid \operatorname{rank}_{z} \widetilde{F}<k\right\}$. Let $G \leqq X$. If $[f](G) \cap$ $S_{b} \neq \varnothing$ for some $b \in N$, then $G$ is called effective (for $\mathfrak{A}$ ). The set of all $b \in N$ to which $F$ is adapted at every point of ${ }^{\prime} G=P^{-1}(G)$ is denoted by $N_{G, f}$. Let $\widetilde{G}=h^{\prime-1}\left({ }^{\prime} G\right)$. Then $N_{G, f}=N-\widetilde{F}(\widetilde{G} \cap D)$. Hence if $G$ is compact, $N_{G, f}$ is open. Let $G_{f}^{(0)}$ be the set of all $x \in G$ such that $F$ is truly adapted to some $b \in N$ at some $w \in P^{-1}(x)$.

Lemma 1.2. Assume $f$ is almost adapted to 2 . Then (i) for all $G \leqq X, N-N_{G, f}$ has zero (Hausdorff) $2 k$-measure; (ii) an open set $G \subseteq X$ is effective iff $G_{f}^{(0)} \neq \varnothing$; (iii) for every branch $X_{j}$ of $X$, there exists $x_{j} \in X_{j} \cap X^{0}$ such that $f_{0}$ is truly adapted to some point of $N$ at $x_{j}$.

Proof. By $[1,1.24], \widetilde{F}(D)$ is almost thin of dimension $k-2$. Since $N-N_{G, f} \subseteq \widetilde{F}(D)$, assertion (i) follows. Assume $G \subseteq X$ is open. If $G$ is effective there exists $w \in^{\prime} G$ such that $F(w) \in h(M)$. Then $G_{f}^{(0)} \supseteq P\left(h^{\prime}(\widetilde{G}-D)\right) \neq \varnothing$, since $D$ is thin analytic in ' $\widetilde{X}([1,1.16])$. The converse is trivial. Now (iii) is a consequence of (ii). 
LEMMA 1.3. Assume every effective branch $X_{j}$ of $X$ contains a point $x_{j} \in X^{0}$ such that for every branch $B$ of $W=h^{-1}\left(f\left(x_{j}\right)\right), f_{0}$ is truly adapted to some point of $\pi(B)$ at $x_{j}$. Then $f$ is almost adapted to $\mathscr{H}$.

Proof. Let $\left(f_{0}^{\prime}, h_{0}^{\prime}\right)$ be the fiber product of $\left(f_{0}, h\right)$. Take an arbitrary branch $A$ of $\widetilde{X}^{0}$. The map $h_{0}^{\prime} \mid A: A \rightarrow X^{0}$ is proper of pure maximal rank. Hence the image $A^{\prime}=h_{0}^{\prime}(A)$ is a branch of $X^{0}$ ([1, 1.27]). Let $\left\{B_{i}\right\}$ be the family of branches of $W$. Since $A^{\prime}$ is the intersection of $X^{0}$ and an effective branch $X_{j}$ of $X$, it contains a point $x_{j}$ at which $f_{0}$ is truly adapted to a point of $\pi\left(B_{i}\right)$ for every $B_{i}$. Also, $\left(h_{0}^{\prime}\right)^{-1}\left(x_{j}\right) \cap A \neq \varnothing$ and contains a branch of the form $\left(f_{0}^{\prime}\right)^{-1}\left(B_{i}\right) \cap\left(h_{0}^{\prime}\right)^{-1}\left(x_{j}\right)$. Hence there exists $z \in A$ with $\operatorname{rank}_{z} \widetilde{f}_{0} \mid A=k$. It follows that $\widetilde{F}$ has strict rank $k$.

COROLLARY 1.4. Let $\mathfrak{A}$ be a st. adm. family defined by $Y \stackrel{h}{\leftarrow} M \stackrel{\pi}{\rightarrow} N$. Assume $Y$ is nonsingular, connected and simply connected, and $M$ is irreducible. Let $f: X \rightarrow Y$ be a meromorphic map such that for every branch $X_{j}$ of $X$, there is a point $x_{j} \in X_{j}-I_{f}$ at which $f_{0}$ is truly adapted to a point of $N$. Then $f$ is almost adapted to $\mathfrak{A}$.

Proof. By $[27,1.3], h^{-1}(y)$ is irreducible for all $y \in Y$. Apply Lemma 1.3.

2. The Crofton formula and the F.M.T. Let $X$ be a complex space of dimension $m>0$. Let $A_{k}^{p}(X)$, resp. $A_{k}^{q, r}(X)$, denote the set of all differential forms of class $C^{k}$ and degree $p$, resp. bidegree $(q, r)$ on $X$. A form $\zeta \in A_{0}^{p, p}(X)$ is said to be nonnegative ( $\left.\geqq 0\right)$ iff for any holomorphic map $\alpha$ of a nonvoid open set $U \subseteq C^{p}$ into $X, \alpha^{*} \zeta \geqq 0$ on $U$; $\zeta$ is said to be strictly nonnegative ( $\gg 0)$ iff $\zeta \wedge \eta \geqq 0$ for all nonnegative forms $\eta$ on $X$. The form $\zeta$ is said to be positive at $a \in X$ iff $\zeta$ has a positive extension into a local embedding space of $X$ at $a$. Also, $\zeta$ is said to be simi-positive on $X$ iff it is positive outside a thin analytic subset of $X$.

LEMMA 2.1. If $\zeta \in A_{0}^{p, p}(X)$ is nonnegative on an open, dense subset of $X$, then $\zeta$ is nonnegative on $X$.

Proof. Take arbitrary $\xi_{j} \in A_{0}^{1,0}(X), j=1, \cdots, m-p$. Then the form $\eta=i^{m-p} \zeta \wedge \xi_{1} \wedge \bar{\xi}_{1} \wedge \cdots \wedge \xi_{m-p} \wedge \bar{\xi}_{m-p}$ is $\geqq 0$ on an open, dense subset of $X$. By continuity, $\eta \geqq 0$ on $X_{\text {reg }}$ (the manifold of regular points of $X)$. It follows that $\eta \geqq 0$ (hence also $\zeta \geqq 0$ ) on $X([28, \S 4.2]$ ).

Let $M, M^{\prime}$ be complex spaces and $h: M \rightarrow M^{\prime}$ a holomorphic map. If $h$ is proper and locally trivial at every point of $M$, then the fiber 
integration operator $h_{*}$ exists, which associates to each $\zeta \in A_{l}^{p, p}(M)$, $p \geqq r=$ fiber $\operatorname{dim}$. $h$, a form $h_{*} \zeta \in A_{l}^{p-r, p-r}\left(M^{\prime}\right)([28, \S 8.2])$. Moreover, if $\zeta$ is $\geqq 0$, resp. $\gg 0$, then so is $h_{*} \zeta$ ([ibid.]). If $h$ has strict rank $n=\operatorname{dim} M^{\prime}$ and if $\zeta^{\prime}$ is $\gg 0$ on $M^{\prime}$, then $h^{*} \zeta^{\prime} \gg 0$ on $M$ ([ibid.]).

Assume $Y \stackrel{h}{\leftarrow} M \stackrel{\pi}{\rightarrow} N$ defines an admissible family $\mathfrak{A}$ in $Y$. Then the linear map $\Psi=h_{*} \pi^{*}: A_{l}^{p, p}(N) \rightarrow A_{l}^{p-k+s, p-k+s}(Y)$ exists if $p \geqq k-s$. Assume $f: X \rightarrow Y$ is a meromorphic map almost adapted to $\mathfrak{A}$.

LEMMA 2.2. Let $\xi, \xi^{\prime} \in A_{0}^{p, p}(N)$ be real forms where $p=k$ or $k-1$. Let $\xi_{Y}=\Psi(\xi)$. (1) If $\xi \geqq 0$, then $f^{*} \xi_{Y} \gg 0$ on any open subset of $X^{0}$. (2) Assume $N$ is compact and $\xi>0$. Then there is a constant $C>0$ such that if $\zeta \in A_{0}^{r, r}(W)$ is $\geqq 0$ on an open subset $W$ of $X, \zeta \wedge f^{*} \xi_{Y}^{\prime} \leqq$ $C \zeta \wedge f^{*} \xi_{Y}$ on $W$.

Proof. Observe that $\xi \gg 0$ on $N$. Since $\widetilde{F}$ has strict rank $k$, fiber integration yields $F^{*} \xi_{Y} \gg 0$ on ' $X$ ([ibid.]). This implies $f^{*} \xi_{Y} \gg 0$ on any open subset of $X^{0}([28,4.2 .5]$.$) . To prove (2), let C>0$ be a constant such that $\xi^{\prime} \leqq C \xi$. Then $C \xi-\xi^{\prime} \gg 0$. Hence $\zeta \wedge f^{*} \xi_{Y}^{\prime} \leqq$ $C \zeta \wedge f^{*} \xi_{Y}$ on $W$.

THEOREM 2.3 (Crofton Formula). Let $\zeta \in A_{0}^{2(m-s)}(X)$ and $\omega \in A_{0}^{2 k}(N)$. Assume $G \subseteq X$ is open and $K=\bar{G} \cap \operatorname{supp} \zeta$ is compact. Define ' $\zeta=$ $P^{*} \zeta,{ }^{\prime} G=P^{-1}(G)$, and

$$
N_{f}\left(G, S_{b}, \zeta\right)=\int_{F^{-1}\left(S_{b}\right) \cap^{\prime} G} \nu_{F}^{b / \zeta} \quad\left(b \in N_{K, f}\right) .
$$

Then $N_{f}\left(G, S_{b}, \zeta\right)$ is measurable on $N$ and

$$
\int_{N} N_{f}\left(G, S_{b}, \zeta\right) \omega=\int_{G} \zeta \wedge f^{*} \omega_{Y}
$$

REMARKS 1. The intersection multiplicity $\nu_{F}^{b}$ of $F$ with $S_{b}$ is included because of its appearance in the F.M.T. For the definition and properties of the multiplicity, see [28].

2. It can be shown that for almost all $S_{b} \in \mathfrak{A}$,

$$
N_{f}\left(G, S_{b}, \zeta\right)=\int_{f_{0}^{-1}\left(S_{h}\right) \cap G} \zeta \text {. }
$$

Proof. Since

$$
\int_{G \cap X^{0}} \zeta \wedge f_{0}^{*} \omega_{Y}=\int_{{ }_{G}}{ }^{\zeta} \zeta \wedge F^{*} \omega_{Y}
$$

exists, the measurable form $\zeta \wedge f^{*} \omega_{Y}$ is integrable over $G$. Moreover, it suffices to consider the case where $f$ is holomorphic. 


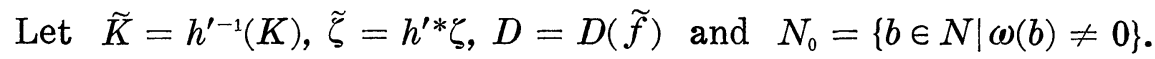
Then

$$
\begin{aligned}
\int_{G} \zeta \wedge f^{*} \omega_{Y} & =\int_{K} \zeta \wedge h_{*}^{\prime}\left(f^{*} \pi^{*} \omega\right) \quad([28, \S 8.2]) \\
& =\int_{\widetilde{K}-D} \tilde{\zeta} \wedge \tilde{f}^{*} \omega \\
& =\int_{N_{0}}\left(\int_{\tilde{f}^{-1}(b) \cap(\widetilde{K}-D)} \nu_{\tilde{f}} \tilde{\zeta}\right) \omega \quad([28,5.2 .1]) \\
& =\int_{N_{K}, f}\left(\int_{\tilde{f}-1(b) \cap \tilde{K}} \nu_{\tilde{f}} \tilde{\zeta}\right) \omega \\
& =\int_{N} N_{f}\left(G, S_{b}, \zeta\right) \omega .
\end{aligned}
$$

LeMma 2.4. Let $\omega \in A_{0}^{2 k}(N)$ be semi-positive. Then (1) $\omega_{Y} \not \equiv 0$ on $Y$. (2) Let $f$ be as above and $\zeta \in A_{0}^{m-s, m-s}(X)$. If $\zeta$ is semi-positive on an effective open set $G \leqq X$, then $\zeta \wedge f^{*} \omega_{Y} \not \equiv 0$ on $G^{0}=G \cap X^{0}$.

Proof. Observe that the identity map of $Y$ is almost adapted to $\mathfrak{A}$ and positive form $\zeta^{\prime} \in A_{0}^{n-s, n-s}(Y)(n=\operatorname{dim} Y)$ exists. Hence (2) implies (1).

To prove (2), let $R$ be a thin analytic subset of $G$ such that $\zeta>0$ on $G-R$. The open set $\left({ }^{\prime} G\right)_{f}^{(0)}$ contains a point $x \in P^{-1}\left(G^{0}\right)$. Therefore it may be assumed w.l.o.g. that $f$ is holomorphic. Let $w \in \widetilde{X}$ such that $f$ is adapted to $a=\widetilde{f}(w)$ at $x=h^{\prime}(w) \in G$. Let $G_{1} \Subset G, Q \subseteq N$ be neighborhoods of $x$, resp. $a$, such that $f^{-1}\left(S_{b}\right) \cap G_{1}$ has pure codimension $s$ for all $b \in Q$. Then $\widetilde{f}\left(\widetilde{G}_{1}\right)$ constains a nonvoid open subset $Q_{1}$ of $Q$. Define $V_{b}=\widetilde{f}^{-1}(b) \cap \widetilde{G}_{1}$ for $b \in Q$, and $\widetilde{R}=h^{\prime-1}(R)$. According to $[1,1.26]$, the set $T=\left\{b \in Q \mid V_{b} \cap \widetilde{R}\right.$ is not thin in $\left.V_{b}\right\}$ is almost thin in $Q$. Since $h^{\prime}$ maps $\widetilde{f}^{-1}(b)$ homeomorphically onto $f^{-1}\left(S_{b}\right)$, there is an open set $H_{b} \subseteq G_{1}$ such that $H_{b} \cap f^{-1}\left(S_{b}\right)=h^{\prime}\left(V_{b}\right)$ (for each $b \in Q)$. Let $Z_{b}=\left(H_{b}-R\right) \cap f^{-1}\left(S_{b}\right)$. Then

$$
\begin{aligned}
\int_{G_{1}} \zeta \wedge f^{*} \omega_{Y} & =\int_{N} N_{f}\left(G_{1}, S_{b}, \zeta\right) \omega \\
& \geqq \int_{Q-T}\left(\int_{V_{b}-\tilde{R}} \nu_{\tilde{f}}^{\widetilde{\zeta}}\right) \omega \\
& \geqq \int_{Q_{1}-T}\left(\int_{Z_{b}} \nu_{f}^{3} \zeta\right) \omega>0 .
\end{aligned}
$$

Let $f: X \rightarrow Y$ be a meromorphic map. Relative to a family of subvarieties in $Y$, say $\mathfrak{A}=\left\{S_{b}\right\}_{b \in N}$, the so called First Main Theorem for $f$ measures the difference between the valence of $S_{b}$ and its mean value on $N$. The theorem requires the existence of certain differential forms $\left\{\Lambda_{b}\right\}_{b \in N}$ (where $\Lambda_{b}$ is singular on $S_{b}$ ) with special properties. 
For instance, if $\mathfrak{A}=\mathfrak{A}_{0 q}$ (the family of projective $q$-planes in $\boldsymbol{P}(V)$ ), the explicitly constructed Chern-Levine forms ([4] [22]) suffice for this purpose. In general, if $\mathfrak{A}$ satisfies $\left(\mathrm{A}_{1}\right)-\left(\mathrm{A}_{4}\right)$, the forms $\Lambda_{b}$ may be obtained by fiber integration from a singular potential $\lambda$ on $N$. The latter means that $\lambda$ is a set of forms $\left\{\lambda_{b}\right\}_{b \in N}$ depending infinitely smoothly on $b$ such that (i) $\lambda_{b}$ is $\geqq 0, C^{\infty}$ on $N-\{b\}$ of bidegree $(k-1, k-1)$; (ii) $\lambda_{b}$ is singular at $b$ as described in [23, p. 55, (2)-(3)] ([28,7.2.1] if $N$ is singular); (iii) $d d^{c} \lambda_{b}$ extends to a nonnegative, $C^{\infty}$ form on $N$ independent of $b$. For a compact Kähler manifold the existence of such forms was proved by Wu [31, I, II], Hirschfolder [14] (see also [13]), and Stoll [23]. By the method of elliptic operators, Dektyarev [8] [9] constructed similar forms without requiring the Kähler condition. Actually, combining [8] and [23], the following can be proved:

THEOREM 2.5. Assume $N$ is a connected, compact complex manifold of dimension $k>0$. Let $\omega$ be a volume form on $N$ normalized so that $\int_{N} \omega=1$. Then there exists a singular potential $\lambda=\left\{\lambda_{b}\right\}_{b \in N}$ such that $d d^{c} \lambda_{b}=\omega$ on $N-\{b\}$.

Proof. By $[23,5.3]$, there exist differential forms $\left\{\lambda_{b}^{*}\right\}_{b \in N}$ depending infinitely smoothly on $b$ such that $\lambda_{b}^{*}$ satisfies the above conditions (i)-(ii), and for some $C^{\infty} g: N \times N \rightarrow \boldsymbol{R}$,

$$
d d^{c} \lambda_{b}^{*}=g_{b} \omega \quad \text { on } \quad N-\{b\} \text {. }
$$

(Here $g_{b}(x)=g(x, b)$ if $x \in N$.) Take a positive form $\xi \in A_{\infty}^{k-1, k-1}(N)$. Consider the linear operator $E$ on the space $\mathscr{C}^{\infty}(N)$ of real-valued $C^{\infty}$-functions such that for $u \in \mathscr{C}^{\infty}(N)$,

$$
(E u) \omega=d d^{c}(u \xi) \text {. }
$$

The adjoint operator $E^{*}$ is given by

$$
\left(E^{*} u\right) \omega=d d^{c} u \wedge \xi \text {. }
$$

Then $E$ and $E^{*}$ are elliptic. By the maximum principle, the kernel of $E^{*}$ consists of constant functions. The residue theorem ([23, 6.4]) applied to the identity map of $N$ gives $\int_{N} g_{b} \omega=1$ for $b \in N$. Hence the equation

$$
E u=1-g_{b}
$$

has a solution $u_{b} \in \mathscr{C}^{\infty}(N)$ depending infinitely smoothly on $b$ (see, for example, $[15,10.5 .3][19])$. According to [8, p. 961], the form 
$\xi$ can be chosen so that $d d^{c} \xi=0$. Hence for some positive constant $C, \lambda_{b}=\left(C+u_{b}\right) \xi+\lambda_{b}^{*}$ defines a set of forms with all required properties.

If $G$ is an open subset of the complex space $X$, there exists a maximal open subset $d G$ of $\partial G \cap X_{\text {reg }}$ such that $d G$ is a smooth, (oriented) $C^{\infty}$-boundary manifold of $G$ in $X_{\text {reg. }}$ A relatively compact open set $G \cong X$ is called a Stokes domain iff $\partial G$ has locally finite (Hausdorff) $(2 m-1)$-measure and $\partial G-d G$ has zero $(2 m-1)$-measure. A bump $(g, G, \psi)$ in $X$ is given by Stokes domains $G$ and $g$ in $X$ with $\varnothing \subseteq g \Subset G$ and a continuous function $\psi: X \rightarrow R[0, \infty)$ such that (i) $\operatorname{supp} \psi \subseteq \bar{G}$; (ii) $\psi \mid \bar{G}-g$ is of class $C^{2}$; (iii) $\psi|\bar{g}=\operatorname{Max} \psi| \bar{G}>0$ (if $g \neq \varnothing$ ).

THEOREM 2.6 (F.M.T.). Let $f: X \rightarrow Y$ be a meromorphic map of a complex space $X$ of dimension $m>0$ into a complex space $Y$. Assume $\mathfrak{A}=\left\{S_{b}\right\}_{b \in N}$ is admissible in $Y$ with $q=m$-codim $\mathfrak{A} \geqq 0$. Assume $\omega \in A_{\infty}^{2 k}(N)$ is nonnegative and $\lambda=\left\{\lambda_{b}\right\}_{b_{b N}}$ is a singular potential with $d d^{c} \lambda_{b}=\omega$. Define $\Lambda_{b}=\Psi\left(\lambda_{b}\right)$ on $Y-S_{b}$ and $\Omega=\Psi(\omega)$ on $Y$. Assume $\chi \in A_{1}^{q, q}(X)$ is closed, strictly nonnegative. Let $(g, G, \psi)$ be a bump in $X$, and $K=\bar{G} \cap \operatorname{supp} \chi$. Then for every $b \in N_{K, f}$,

$$
T_{f}(G)-N_{f}(G, b)=m_{f}(\Gamma, b)-m_{f}(\gamma, b)-D_{f}(G, b) .
$$

Here

$$
\begin{aligned}
N_{f}(G, b) & =\int_{F^{-1}\left(S_{b}\right) \cap^{\prime} \mathcal{H}^{\prime}} \boldsymbol{\nu}^{b^{\prime} \psi^{\prime} \chi} & & \text { (valence) } \\
m_{f}(\Gamma, b) & =\int_{\Gamma} f^{*} \Lambda_{b} \wedge d^{\perp} \psi \wedge \psi \geqq 0 & & \text { (exterior proximity) } \\
m_{f}(\gamma, b) & =\int_{\gamma} f^{*} \Lambda_{b} \wedge d^{\perp} \psi \wedge \chi \geqq 0 & & \text { (interior proximity) } \\
T_{f}(G) & =\int_{G} \psi f^{*} \Omega \wedge \chi & & \text { (characteristic) } \\
D_{f}(G, b) & =\int_{G-\vec{g}} f^{*} \Lambda_{b} \wedge d d^{\perp} \psi \wedge \chi & & \text { (deficit) }
\end{aligned}
$$

are continuous functions of $b$ on $N_{K, f}$; 'G,' $\psi$, resp. ' $\chi$, denotes its lifting to ' $X ; \Gamma=\partial G, \gamma=\partial g$, and $d^{\perp}=i(\bar{\partial}-\partial)=-d^{c}$.

REMARKS 1. If, in the F.M.T., $f$ is almost adapted to $\mathfrak{A}$, the hypothesis " $\chi \gg 0$ " can be weakened to " $\chi \geqq 0$ in a neighborhood of $\bar{G}-g$ ". 2. The theorem was proved in [28] for a holomorphic map; the case of a meromorphic map is an easy consequence.

3. Integral averages. Some general assumptions shall be stated here for later reference. 
( I ) $X$ is a complex space of dimension $m>0$ with at least one noncompact branch.

(II) $\mathfrak{A}=\left\{S_{b}\right\}_{b \in N}$ is admissible in a complex space $Y$, where $N$ is compact, connected and nonsingular. Let $k=\operatorname{dim} N, s=\operatorname{codim} S_{b}$, and $q=m-s$.

(III) $f: X \rightarrow Y$ is a meromorphic map almost adapted to $\mathfrak{A}$.

(IV-a) $\omega \in A_{\infty}^{2 k}(N)$ is semi-positive, normalized so that $\int_{N} \omega=$ $1 ;\left\{\lambda_{b}\right\}_{b \in N}$ is a singular potential with $d d^{c} \lambda_{b}=\omega$. Define $\Lambda_{b}=\Psi\left(\lambda_{b}\right)$, $\Omega=\omega_{Y}$.

(IV-b) $\omega_{N, 1}$ is the fundamental form on $N$ of a Hermitian metric normalized so that $\int_{N} \omega_{N, 1}^{k}=1$. Define $\Omega_{l}=\left(\omega_{N, 1}^{k-s+l}\right)_{Y}, 0 \leqq l \leqq s$.

(V) If $q=0$, let $\chi=1$, if $q>0$, assume $\chi \in A_{2}^{p, q}(X)$ is closed, nonnegative.

(VI) Either $\chi$ is semi-positive on an effective open subset of $X$ or $\chi \wedge f^{*} \Omega \neq 0$ on $X^{0}$.

Assume (I)-(IV-a). For a measurable function $u$ on $N$, define $I(u)=\int_{N} u \omega$ (if the integral exists). By [23,6.3], the integral average $\Lambda(y)=\int_{N} \omega(b) \otimes \lambda_{b}(y), y \in N$, defines a nonnegative form $\Lambda \in A_{0}^{k-1, k-1}(N)$.

LeMma 3.1. Let $G \subseteq X$ be a relatively compact open set. Assume $\zeta \in A_{0}^{q+1, q+1}(X)$. Then the integral

$$
D_{f}(G ; \zeta)=\int_{G} f^{*} \Lambda_{Y} \wedge \zeta
$$

exists and

$$
I\left(\int_{G} f^{*} \Lambda_{b} \wedge \zeta\right)=D_{f}(G ; \zeta)
$$

Proof. The existence of $D_{f}(G ; \zeta)$ follows from the continuity of $F^{*} \Lambda_{Y} \wedge^{\prime} \zeta$ on ' $G$. By [28, 7.2.2 and $\left.\S 8.2\right]$, the integral $\int_{G} f^{*} \Lambda_{b} \wedge \zeta$ is a continuous function of $b$ on $N_{\bar{G}, f}$. Also by [28, 9.1$], F^{*} \Lambda_{b} \gg 0$ on ' $X-F^{-1}\left(S_{b}\right)$. Let $\tilde{\zeta}=h^{\prime *}(' \zeta)$. If $\zeta \geqq 0$ on $G$, then

$$
\begin{aligned}
I\left(\int_{G} f^{*} \Lambda_{b} \wedge \zeta\right) & =\int_{N}\left(\int_{\widetilde{G}} \widetilde{F}^{*} \lambda_{b} \wedge \tilde{\zeta}\right) \omega \\
& =\int_{G^{\prime}} F^{*} \Lambda_{Y} \wedge^{\prime} \zeta .
\end{aligned}
$$

If $\zeta$ is real, there exists a positive form $\eta \in A_{0}^{q+1, q+1}(X)$ such that $\eta+\zeta>0$ on $\bar{G}$. Then (3.1) holds for $\eta$ and $\eta+\zeta$, hence also for $\zeta$. 
If $\zeta$ is complex-valued, a splitting into real and imaginary parts yields the result.

LEMMA 3.2. Let $\chi \in A_{2}^{q, q}(X)$ and $G \leqq X$ be a Stokes domain. Assume $\psi: X \rightarrow \boldsymbol{R}$ is of class $C^{2}$ with $\psi \mid \partial G=0$, and, for some neighborhood $W$ of $\partial G$, either $\psi \mid W \cap G \geqq 0$ or $\psi \mid W-G \geqq 0$. Then

$$
\begin{aligned}
I\left(\int_{d G} f^{*} \Lambda_{b} \wedge d^{c} \psi \wedge \chi\right) & =\int_{d G} f^{*} \Lambda_{Y} \wedge d^{c} \psi \wedge \chi \\
& =D_{f}\left(G ; d d^{c}(\psi \chi)\right) .
\end{aligned}
$$

Proof. For each $b \in N_{\bar{G}, f}$, it was proved in [30] that the following residue formula holds:

$$
\int_{G} \psi f^{*} \Omega \wedge \chi-\int_{G} f^{*} \Lambda_{b} \wedge d d^{c}(\psi \chi)=N_{f}\left(G, S_{b}, \psi \chi\right)-\int_{d G} f^{*} \Lambda_{b} \wedge d^{c} \psi \wedge \chi .
$$

Therefore (3.1) and the Crofton Formula yield

$$
I\left(\int_{d G} f^{*} \Lambda_{b} \wedge d^{c} \psi \wedge \chi\right)=D_{f}\left(G ; d d^{c}(\psi \chi)\right) .
$$

Now assume $\chi \geqq 0$ in a neighborhood of $\bar{G}$. Let $\Gamma=\partial G,{ }^{\prime} \Gamma=P^{-1}(\Gamma)$, $\widetilde{\Gamma}=h^{\prime-1}(' \Gamma)$, etc. By $[23,3.2$ and AII, 4.11, 4.6],

$$
\begin{aligned}
I\left(\int_{d G} f^{*} \Lambda_{b} \wedge d^{c} \psi \wedge \chi\right) & =\int_{N}\left(\int_{\Gamma} F^{*} \Lambda_{b} \wedge d^{c \prime} \psi \wedge^{\prime} \chi\right) \omega \\
& =\int_{N}\left(\int_{\widetilde{T}} \widetilde{F}^{*} \lambda_{b} \wedge d^{c} \widetilde{\psi} \wedge \tilde{\chi}\right) \omega \\
& =\int_{\Gamma^{\prime}} F^{*} \Lambda_{Y} \wedge d^{c} \psi \wedge \wedge^{\prime} \chi \\
& =\int_{\Gamma} f^{*} \Lambda_{Y} \wedge d^{c} \psi \wedge \chi .
\end{aligned}
$$

The general case follows the same way as in Lemma 3.1.

Assume (I)-(IV-a) and (V). Assume $(g, G, \psi)$ is a bump in $X$. Let $T_{f}, N_{f}, m_{f}$, and $D_{f}$ denote the associated value distribution functions. Let $\hat{\psi}$ be a $C^{2}$-extension of $\psi$ on $X$. Then Lemma 3.2 applied to $\hat{\psi}$, resp. $(\hat{\psi} \mid g)-\hat{\psi}$, shows that the mean proximities $m_{f}(\Gamma)=$ $I\left(m_{f}(\Gamma, b)\right)$ and $m_{f}(\gamma)=I\left(m_{f}(\gamma, b)\right)$ exist. Moreover, with $G_{\mu}=G$ or $g$,

$$
m_{f}\left(\partial G_{\mu}\right)=\int_{d G_{\mu}} f \Lambda_{Y} \wedge d^{\perp} \psi \wedge \chi=D_{f}\left(G_{\mu} ; d^{c} d \hat{\psi} \wedge \chi\right) .
$$

Hence

$$
m_{f}(\Gamma)-m_{f}(\gamma)=I\left(D_{f}(G, b)\right)
$$


4. Equidistribution theorems. Let $X$ be a complex space of dimension $m>0$. A semi-exhaustion function of $X$ is an upper semicontinuous map $\varphi: X \rightarrow \boldsymbol{R}_{-\infty}=\boldsymbol{R} \cup\{-\infty\}$ such that the half spaces $X[r]=\{x \in X \mid \phi(x) \leqq r\}$ are compact for all $r \geqq 0$. An exhaustion function of $X$ is a semi-exhaustion $\varphi: X \rightarrow \boldsymbol{R}_{-\infty}$ which is $C^{\infty}$ outside a compact set.

Let $\varphi$ be a semi-exhaustion function of $X$. If $r>r^{\prime} \geqq-\infty$, define $X(r)=\{x \in X \mid \varphi(x)<r\}, X\left[r^{\prime}, r\right)=X(r)-X\left(r^{\prime}\right), X\left(r^{\prime}, r\right)=X(r)-$ $X\left[r^{\prime}\right]$, etc. Assume $U: \boldsymbol{R}\left[r_{0}, r\right) \rightarrow \boldsymbol{R}$ is absolutely continuous and let $u=U^{\prime}$. For $r>r^{\prime} \geqq r_{0}$, define

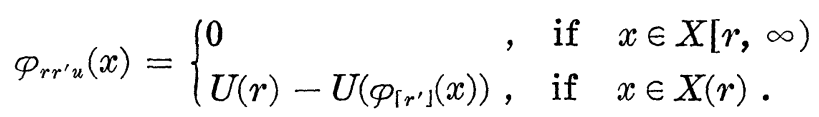

Here $\varphi_{\left[r^{\prime}\right]}(x)=\operatorname{Max}\left(r^{\prime}, \varphi(x)\right)$. Define $U_{\varphi}=U \circ \varphi$.

Lemma 4.1. (Cf. [23, 8.3] [25, 10.6].) Assume $\zeta$ is a locally integrable $2 m$-form on $X$. Take $a_{0} \in R[-\infty, r)$. Define

$$
v(t)=\int_{X\left[a_{0}, t\right)} \zeta, \quad v[t]=\int_{X\left[a_{0}, t\right]} \zeta \quad\left(t>a_{0}\right) .
$$

Then if $r>r^{\prime} \geqq \operatorname{Max}\left(a_{0}, r_{0}\right)$,

$$
\begin{aligned}
\int_{X\left[a_{0}, \infty\right)} \varphi_{r r^{\prime} u} \zeta & =\int_{r^{\prime}}^{r} v(t) u(t) d t \\
& =U(r) v(r)-U\left(r^{\prime}\right) v\left[r^{\prime}\right]-\int_{X\left(r^{\prime}, r\right)} U_{\varphi} \zeta .
\end{aligned}
$$

Proof. Observe that $\varphi_{r r^{\prime} u}$ is bounded, measurable on $X$. W.l.o.g. assume $\zeta$ is real. There exist nonnegative, integrable forms $\zeta_{j}(j=1,2)$ on $X_{\text {reg }}[r]$ such that $\zeta_{1}-\zeta_{2}=\zeta$. Hence it may be assumed that $\zeta \geqq 0$ on $X_{\text {reg }}[r]$. Likewise assume $u \geqq 0$. Let $C(x, t)=1$ if $\varphi(x)<t$, and $C(x, t)=0$ if $\varphi(x) \geqq t$. Then

$$
\begin{aligned}
\int_{X\left[a_{0}, \infty\right)} \varphi_{r r^{\prime} u} \zeta & =\int_{X\left[a_{0}, r\right)}\left(\int_{\varphi_{\left[r^{\prime}\right](x)}^{r}}^{r} u(t) d t\right) \zeta(x) \\
& =\int_{r^{\prime}}^{r}\left(\int_{X\left[a_{0}, r\right)} C(x, t) \zeta(x)\right) u(t) d t \\
& =\int_{r^{\prime}}^{r} v(t) u(t) d t .
\end{aligned}
$$

On the other hand,

$$
\begin{aligned}
\int_{X\left[a_{0}, \infty\right)} \varphi_{r r^{\prime} u} \zeta & =\int_{X\left[a_{0}, r\right)}\left[U(r)-U\left(\varphi_{\left[r^{\prime}\right]}(x)\right)\right] \zeta(x) \\
& =U(r) v(r)-U\left(r^{\prime}\right) v\left[r^{\prime}\right]-\int_{X\left(r^{\prime}, r\right)} U_{\varphi} \zeta .
\end{aligned}
$$


Assume (I) and let $\varphi: X \rightarrow \boldsymbol{R}_{-\infty}$ be an exhaustion function. Call $r>-\infty \phi$-admissible if $X(r)$ is a Stokes domain. A strictly increasing sequence $\left\{r_{j}\right\}_{j=1}^{\infty}$ is said to be $\varphi$-admissible if each $r_{j}$ is $\varphi$-admissible and $r_{j} \rightarrow \infty$. The set of non- $\phi$-admissible values $>r^{\prime}$ has measure zero in $R\left[r^{\prime}, \infty\right)$ for large $r^{\prime}$ (cf. [28, 7.1.6]). If $\varphi$ is $C^{\infty}$ on $X$, this is true for arbitrary $r^{\prime}$.

LEMMA 4.2. Let $L(\varphi)=d d^{\circ} \varphi$ be the Levi form of $\varphi$ on $X-X\left[r_{0}\right]$ (where $\varphi$ is $C^{\infty}$ ). Assume $U: R\left[r_{0}, \infty\right) \rightarrow \boldsymbol{R}$ is of class $C^{2}$ and $u=U^{\prime}$. Let $\zeta \in A_{2}^{m-1, m-1}(X)$. (1) If $r, r^{\prime}$ are $\varphi$-admissible with $r>r^{\prime}>r_{0}$, then

$$
\begin{aligned}
& u(r) \int_{d X(r)} d^{c} \varphi \wedge \zeta-u\left(r^{\prime}\right) \int_{d X\left(r^{\prime}\right)} d^{c} \varphi \wedge \zeta \\
& \quad=\int_{X\left(r^{\prime}, r\right)} L\left(U_{\varphi}\right) \wedge \zeta+\int_{r^{\prime}}^{r}\left(\int_{X(t)} d d^{c} \zeta\right) u(t) d t .
\end{aligned}
$$

(2) Assume $\varphi: X \rightarrow \boldsymbol{R}$ is $C^{\infty}$ and $\zeta$ is $d^{c} d$-closed. Then for all $r>$ $r^{\prime}>r_{0}$

$$
u(r) \int_{X(r)} L(\varphi) \wedge \zeta-u\left(r^{\prime}\right) \int_{X\left(r^{\prime}\right)} L(\varphi) \wedge \zeta=\int_{X\left[r^{\prime}, r\right)} L\left(U_{\varphi}\right) \wedge \zeta .
$$

Proof. By Lemma 4.1, if $r, r^{\prime}$ are $\phi$-admissible with $r>r^{\prime}>r_{0}$, then

$$
\begin{aligned}
\int_{r^{\prime}}^{r}\left(\int_{X(t)} d d^{c} \zeta\right) u(t) d t= & U(r) \int_{X(r)} d d^{c} \zeta-U\left(r^{\prime}\right) \int_{X\left[r^{\prime}\right]} d d^{c} \zeta-\int_{X\left(r^{\prime}, r\right)} U_{\varphi} d d^{\circ} \zeta \\
& =\int_{X\left(r^{\prime}, r\right)} d\left(U_{\varphi}\right) \wedge d^{c} \zeta \\
& =\int_{X\left(r^{\prime}, r\right)} d \zeta \wedge d^{c}\left(U_{\varphi}\right) \\
& =u(r) \int_{d X(r)} d^{c} \varphi \wedge \zeta-u\left(r^{\prime}\right) \int_{d X\left(r^{\prime}\right)} d^{c} \varphi \wedge \zeta \\
& -\int_{X\left(r^{\prime}, r\right)} \zeta \wedge L\left(U_{\varphi}\right) .
\end{aligned}
$$

Now assertion (2) follows from (1) by repeated application of the Stokes theorem $([28, \S 7.1])$ and the left-continuity of $v(t)=\int_{X(t)} \chi$ (where $\chi \in A_{\mathrm{loc}}^{2 m}(X)$ ).

Let $\varphi: X \rightarrow \boldsymbol{R}_{-\infty}$ be an exhaustion function. If there exists an increasing $g: \boldsymbol{R}\left(c_{0}, \infty\right) \rightarrow \boldsymbol{R}\left(c_{0} \geqq 0\right)$ of class $C^{1}$ such that

(i) $L(\varphi) \geqq g_{\varphi}^{\prime} d \varphi \wedge d^{c} \varphi$ on $X_{\text {reg }}-X\left[c_{0}\right]$,

(ii) $\left\|e^{-g}\right\|_{c_{0}}^{r} \rightarrow \infty$ as $r \rightarrow \infty$,

then $\varphi$ is called g-convex. If instead of (i),

(i) $L(\varphi) \leqq g_{\varphi}^{\prime} d \varphi \wedge d^{c} \varphi$ on $X_{\mathrm{reg}}-X\left[c_{0}\right]$, 
and (ii) hold, then $\varphi$ is called g-concave.

A $g$-convex exhaustion function may not be logarithmic pseudoconvex. As an example, consider the variety $A=\left\{z \in C^{n} \mid z_{1}+\cdots\right.$ $\left.+z_{n-1}=0\right\}, n>1$. Let $\|z\|^{2}=\Sigma z_{j} \bar{z}_{j}, z \in C^{n}$. Then $\varphi=\log \|z\|^{2}: A \rightarrow$ $\boldsymbol{R}_{-\infty}$ is pseudoconvex (i.e., $g$-convex with $g=$ constant). Let $Z=$ $\left\{z \in C^{n} \mid z_{1}=\cdots=z_{n-1}=0\right\}$ and $\iota: Z \rightarrow A$ be the inclusion. Then

$$
\iota^{*}\left(L(\varphi)-\frac{d \varphi \wedge d^{\circ} \varphi}{\varphi}\right)=\frac{-2 d x_{n} \wedge d y_{n}}{\left|z_{n}\right|^{2}}<0
$$

on $Z-A[0]$ (where $z_{n}=x_{n}+i y_{n}$ ). Hence $\varphi$ is not logarithmic psc. (=log - convex). Similarly, one can construct a $g$-concave exhaustion function which is not pseudoconcave.

Assume $g: \boldsymbol{R}\left(c_{0}, \infty\right) \rightarrow \boldsymbol{R}\left(c_{0} \geqq 0\right)$ is of class $C^{1}$ and $u=e^{-g}$. Let $U_{p}$ be a primitive of $u^{p}$ on $\boldsymbol{R}\left(c_{0}, \infty\right)$ for $p \in \mathbb{Z}[1, m]$. Define

$$
\omega_{u}=u_{\varphi}\left[L(\varphi)-g_{\varphi}^{\prime} d \varphi \wedge d^{c} \varphi\right]
$$

off a compact set, say $X[c], c \geqq c_{0}$. Setting $\chi_{l}=L(\varphi)^{l}$, (4.4) yields

$$
\left(\omega_{u}\right)^{p}=d d^{c}\left(U_{p} \circ \varphi\right) \wedge \chi_{p-1} \quad \text { on } X-X[c] \text {. }
$$

Now assume (I) and the exhaustion $\varphi: X \rightarrow \boldsymbol{R}$ is $C^{\infty}$. Let $\zeta \in$ $A_{2}^{p, p}(X)$ be $d d^{c}$-closed; if $p=0$, set $\zeta=1$. Define

$$
A_{p}^{u}(r)=u(r)^{m-p} \int_{X(r)} \zeta \wedge \chi_{m-p} \quad\left(r>c_{0}\right) .
$$

For $r>r^{\prime}>c,(4.3)$ yields

$$
A_{p}^{u}(r)-A_{p}^{u}\left(r^{\prime}\right)=\int_{X\left[r^{\prime}, r\right)} \zeta \wedge\left(\omega_{u}\right)^{m-p} .
$$

Hence if $\varphi$ is $c . g$-convex $\left(c_{0}=0\right)$ and if $\zeta \geqq 0, A_{p}^{u}(0)=\lim _{r \rightarrow 0+} A_{p}^{u}(r)$ exists and

$$
A_{p}^{u}(r)=A_{p}^{u}(0)+\int_{X(r)} \zeta \wedge\left(\omega_{u}\right)^{m-p} \quad(r>0) .
$$

A c.g-convex exhaustion $\varphi$ of a complex space $X$ is called $g$ quasiparabolic if

$$
\int_{X(r)}\left(\omega_{u}\right)^{m}=o\left(\|u\|_{1}^{r}\right) \quad(r \longrightarrow \infty) .
$$

Consider the following example. Let $M \cong \boldsymbol{P}_{n}$ be a projective variety of dimension $m-1>0$. Let $U$ be the restriction of the universal line bundle (over $\boldsymbol{P}_{n}$ ) to $M$. There is a proper, holomorphic map $\sigma$ of $U$ onto an algebraic set $A$ in $C^{n+1}$ such that $\sigma: U-\sigma^{-1}(0) \rightarrow$ $A-\{0\}$ is biholomorphic. Let $\psi=1+\|z\|^{2}: A \rightarrow \boldsymbol{R}$ and $\varphi=\sigma^{*} \psi: U \rightarrow \boldsymbol{R}$. 
With $g=\log r$ in $(4.4)$, the $(1,1)$-form $\omega_{u}$ is $\geqq 0$ on $U$ and $>0$ on $U-\sigma^{-1}(0)$. Moreover,

$$
\int_{U(r)}\left(\omega_{u}\right)^{m}=\int_{A_{\psi}(r)}\left(L\left(\log \psi^{\prime}\right)\right)^{m}=O(1) \quad(r \longrightarrow \infty) .
$$

Thus the exhaustion $\varphi$ of $U$ is log-quasiparabolic but not parabolic in the sense of [25].

Lemma 4.3. Assume $u, A: \boldsymbol{R}\left[r_{0}, \infty\right) \rightarrow \boldsymbol{R}$ where $u$ is positive, continuous, and $A \geqq 0$. Assume

There exists a continuous $\alpha: \boldsymbol{R}\left[r_{0}, \infty\right) \rightarrow \boldsymbol{R}(0, \infty)$ such that $\|u \alpha\|_{r_{0}} \rightarrow \infty$ and $A / \alpha$ is increasing in $r$.

Then

$$
\frac{\|u A\|_{r_{0}}^{r}}{\|u \alpha\|_{r_{0}}^{r}} \longrightarrow \lim _{r \rightarrow \infty} \frac{A(r)}{\alpha(r)}
$$

Proof. Let $G(r)=\|u \alpha\|_{r_{0}}^{r}$. For all $r>r^{\prime}>r_{0}$,

$$
\frac{A\left(r^{\prime}\right)}{\alpha\left(r^{\prime}\right)}\left[1-\frac{G\left(r^{\prime}\right)}{G\left(r^{\prime}\right)}\right] \leqq \frac{\|u A\|_{r_{0}}^{r}}{G(r)} \leqq \frac{A(r)}{\alpha(r)} .
$$

From this the lemma follows.

Theorem 4.4. Assume (I)-(IV-a), (V)-(VI). Let $\varphi: X \rightarrow \boldsymbol{R}_{-\infty}$ be an exhaustion function. Let $U: \boldsymbol{R}\left[r_{0}, \infty\right) \rightarrow \boldsymbol{R}$ be of class $C^{2}$ with $U^{\prime}=u>0$. For $r>r^{\prime} \geqq r_{0}$, define

$$
\begin{aligned}
N_{f}^{u}\left(r, r^{\prime}, S_{b}\right) & =\int_{r^{\prime}}^{r} N_{f}\left(X(t), S_{b}, \chi\right) u(t) d t, \quad\left(b \in N_{X[r], f}\right) \\
T_{f}^{u}\left(r, r^{\prime}, \Omega\right) & =\int_{X} \varphi_{r r^{\prime} u} f^{*} \Omega \wedge \chi,
\end{aligned}
$$

and for $\varphi$-admissible $r>r_{0}$,

$$
m_{f}^{u}(r)=u(r) \int_{d X(r)} f^{*} \Lambda_{Y} \wedge d^{c} \varphi \wedge \chi
$$

Assume one of the following holds:

(1) $L\left(U_{\varphi}\right) \wedge \chi \geqq 0$ off a compact set, and

$$
m_{f}^{u}(a)=o^{\prime}\left(T_{f}^{u}\left(a, r_{0}, \Omega\right)\right)
$$

over some $\varphi$-admissible sequence $\sigma=\left\{a_{j}\right\}_{j=1}^{\infty}$ (this fact is denoted by "o"”).

(2) $L\left(U_{\varphi}\right) \wedge \chi \leqq 0$ off a compact set and $T_{f}^{u}\left(r, r_{0}, \Omega\right) \rightarrow \infty$. Let 
$\sigma=\left\{a_{j}\right\}$ be any $\Phi$-admissible sequence.

Then there exists a set $N_{\sigma} \subseteq N$ of measure zero such that for every $b \in N-N_{o}$, there is a subsequence $\left\{r_{\mu}\right\} \subseteq \sigma, r_{\mu} \rightarrow \infty$, for which

$$
\lim _{\mu \rightarrow \infty} \frac{N_{f}^{u}\left(r_{\mu}, r_{1}, S_{b}\right)}{T_{f}^{u}\left(r_{\mu}, r_{1}, \Omega\right)}=1
$$

Proof. There exists $a_{l-1} \geqq r_{0}$ such that that on $X-X\left[a_{l-1}\right] \varphi$ is $C^{\infty}$ and $L\left(U_{\varphi}\right) \wedge \chi \geqq 0$ (or $\left.\leqq 0\right)$. Define $N^{[\sigma]}=\bigcap_{j=l}^{\infty} N_{X\left[a_{j}\right], f}$. Let $G_{j}=$ $X\left(a_{j}\right), \psi_{j}=\varphi_{a_{j} a_{l} u}$; associated to the bump $\left(G_{l}, G_{j}, \psi_{j}\right), j>l$, there are the deficit $D_{f}\left(a_{j}, b\right)=D_{f}\left(G_{j}, b\right)$, proximity $m_{f}\left(a_{j}, b\right)=m_{f}\left(\partial G_{j}, b\right)$, etc., for all $b \in N^{[\sigma]}$. Observe that

$$
m_{f}\left(a_{j}, b\right)=u\left(a_{j}\right) \int_{d X\left(a_{j}\right)} f^{*} \Lambda_{b} \wedge d^{c} \varphi \wedge \chi \quad(j \geqq l) .
$$

For $b \in N^{[\sigma]}$, define

$$
\Delta_{f}\left(a_{j}, b\right)=\left|D_{f}\left(a_{j}, b\right)\right|+m_{f}\left(a_{j}, b\right)+m_{f}\left(a_{l}, b\right) \quad(j>l) .
$$

Then it follows from (3.3) and (4.8) that

$$
I\left(\Delta_{f}\left(a_{j}, b\right)\right)=o\left(T_{f}\left(a_{j}\right)\right) \quad(j \longrightarrow \infty) .
$$

Define

$$
\begin{aligned}
& N_{j \mu}=\left\{b \in N^{[\sigma]} \mid \Delta_{f}\left(a_{j}, b\right) \geqq 2^{-\mu} T_{f}\left(a_{j}\right)\right\} \quad(j \geqq \mu>l), \\
& N^{(\mu)}=\bigcap_{j \geqq \mu} N_{j \mu} .
\end{aligned}
$$

Then $N^{(\mu)}$ is measurable, and since

$$
I\left(\Delta_{f}\left(a_{j}, b\right)\right) \geqq 2^{-\mu} T_{f}\left(a_{j}\right) \int_{N(\mu)} \omega,
$$

each $N^{(\mu)}$ has measure zero. Define

$$
N_{\sigma}=\left(N-N^{[\sigma]}\right) \cup \bigcup_{\mu=l+1}^{\infty} N^{(\mu)} .
$$

Then $N_{\sigma}$ has measure zero. For each $b \in N-N_{\sigma}$, there exists a subsequence $\left\{r_{\mu}\right\}_{\mu=1}^{\infty} \leqq \sigma$ with $a_{l} \leqq r_{1}<r_{u} \rightarrow \infty$ such that

$$
\Delta_{f}\left(r_{\mu}, b\right)=o\left(T_{f}\left(r_{\mu}\right)\right)
$$$$
(\mu \longrightarrow \infty)
$$

Hence from this and the F.M.T. the theorem follows.

TheOREm 4.5. Assume (I)-(III), (IV-b), (V). Let $\varphi, U, u$ be the same as in Theorem 4.4. Assume $A(r)=\int_{X(r)} f^{*} \Omega_{s} \wedge \chi \neq 0$ and (4.7) holds for $(u, A)$. Assume $\zeta=L\left(U_{\varphi}\right) \wedge \chi \geqq 0$ off a compact set. 
$I f$

$$
\int_{X\left(r_{0}, r\right)} f^{*} \Lambda_{Y} \wedge \zeta=o^{\prime}\left(T_{f}^{u}\left(r, r_{0}, \Omega_{s}\right)\right)
$$

(over some $\varphi$-admissible sequence $\sigma=\left\{\boldsymbol{r}_{j}\right\}$ ), then $\operatorname{Im}[f]$ intersects almost every $S_{b} \in \mathfrak{A}$ with

$$
\lim _{r \rightarrow \infty}^{\prime} \frac{N_{f}^{u}\left(r, r_{c}, S_{b}\right)}{T_{f}^{u}\left(r, r_{0}, \Omega_{s}\right)}=1 .
$$

Here $r$ runs over a subsequence (of $\sigma) \rightarrow \infty$ depending on $S_{b}$. (2) (Cf. Griffiths-King [12, 5.3].) If $\omega_{N, 1}, \omega_{N, 1}^{\prime}$, are cohomologous Kähler forms and if there exists a positive form $\xi \in A_{0}^{k-1, k-1}(N)$ such that

$$
\int_{X\left(r_{0}, r\right)} f^{*} \xi_{Y} \wedge \zeta=o^{\prime}\left(T_{f}^{u}\left(r, r_{0}, \Omega_{s}\right)\right)
$$

(over $\sigma$ as above), then

$$
\lim _{j \rightarrow \infty} \frac{T_{f}^{u}\left(r_{j}, r_{0}, \Omega_{s}^{\prime}\right)}{T_{f}^{u}\left(r_{j}, r_{0}, \Omega_{s}\right)}=1
$$

Proof. By (4.1) and Lemma 4.3, $T_{f}^{u}\left(r, r_{0}, \Omega_{s}\right) \rightarrow \infty$. (1) Let $r, r^{\prime}$ be $\varphi$-admissible with $r>r^{\prime}>r_{0}$. By (3.1) and (3.3),

$$
\begin{aligned}
\int_{X\left(r_{0}, r\right)} f^{*} \Lambda_{Y} \wedge \zeta & =O(1)+\int_{X\left(r^{\prime}, r\right)} f^{*} \Lambda_{Y} \wedge d^{c} d \varphi_{r r^{\prime} u} \wedge \chi \\
& =O(1)+m_{f}^{u}(r)-m_{f}^{u}\left(r^{\prime}\right) .
\end{aligned}
$$

Thus (4.8) follows from (4.9) and therefore (4.10) holds. (2) Lemma 2.2 and (4.11) yield

$$
\int_{X\left(r_{0}, r\right)} f^{*} \Omega_{s-1} \wedge \zeta=o^{\prime}\left(T_{f}^{u}\left(r, r_{0}, \Omega_{s}\right)\right)
$$

There exists $\eta \in A_{\infty}^{k-1, k-1}(N)$ such that $d d^{c} \eta=\omega_{N, 1}^{\prime k}-\omega_{N, 1}^{k}$. Assume $r, r^{\prime}$ are $\rho$-admissible, $r>r^{\prime}>r_{0}$, and $\zeta \geqq 0$ on $X-X\left[r^{\prime}\right]$. By (4.2),

$$
u(r) \int_{d X(r)} d^{c} \varphi \wedge f^{*} \Omega_{s-1} \wedge \chi=O(1)+\int_{X\left(r_{0}, r\right)} L\left(U_{\varphi}\right) \wedge f^{*} \Omega_{s-1} \wedge \chi .
$$

Observe that on $d X^{0}(r)$,

$$
d^{c} \varphi \wedge f^{*} \eta_{Y} \wedge \chi \leqq \text { const. } d^{c} \varphi \wedge f^{*} \Omega_{s-1} \wedge \chi
$$

$([23,3.2])$. Therefore (4.2) yields 


$$
\begin{aligned}
T_{f}^{u}\left(r, r^{\prime}, \Omega_{s}^{\prime}\right)-T_{f}^{u}\left(r, r^{\prime}, \Omega_{s}\right) \\
\quad=O(1)+u(r) \int_{d X(r)} d^{c} \varphi \wedge f^{*} \eta_{Y} \wedge \chi-\int_{X\left(r^{\prime}, r\right)} f^{*} \eta_{Y} \wedge \zeta \\
\quad \leqq O(1)+\text { const. } \int_{X\left(r_{0}, r\right)} f^{*} \Omega_{s-1} \wedge \zeta .
\end{aligned}
$$

Now (4.12) follows from (4.13).

Corollary 4.6. Assume (I)-(IV-a), (V)-(VI). Assume $\varphi$ is a $C^{\infty}, g$ convex exhaustion function of $X$. If (with $u=e^{-g}$ defined on $\boldsymbol{R}\left[r_{0}, \infty\right)$ )

$$
u(r) \int_{X(r)} f^{*} \Lambda_{Y} \wedge \chi_{1} \wedge \chi=o^{\prime}\left(T_{f}^{u}\left(r, r_{0}, \Omega\right)\right),
$$

then for almost every $S_{b} \in \mathfrak{A}$,

$$
\lim _{r \rightarrow \infty} \frac{N_{f}^{u}\left(r, r_{0}, S_{b}\right)}{T_{f}^{u}\left(r, r_{0}, \Omega\right)}=1
$$

Proof. If $r>r_{0}$ is $\varphi$-admissible, Lemma 3.2 yields

$$
D_{f}\left(X(r) ; \chi_{1} \wedge \chi\right)=\int_{d X(r)} f^{*} \Lambda_{Y} \wedge d^{c} \varphi \wedge \chi
$$

Hence Theorem 4.4 concludes the proof.

Corollari 4.7. Assume (I)-(IV-a), (V)-(VI). Assume $\varphi: X \rightarrow \boldsymbol{R}_{-\infty}$ is a g-concave exhaustion function. Let $\sigma=\left\{r_{j}\right\}$ be an arbitrary $\varphi$-admissible sequence. Then there is a set $N_{\sigma} \cong N$ of measure zero such that (4.14) holds for every $S_{b}$ with $b \in N-N_{o}$.

\section{Proof. Apply Theorem 4.4.}

Let $W \rightarrow Y$ be a holomorphic vector bundle of fiber dimension $p \geqq 1$ over a complex space $Y$. Assume $\Gamma(Y, W)$ contains an ample linear subspace $V$ (see [17]) of dimension $n+1 \geqq 2$. Take $q \in$ $Z[0, n-p]$. For $b \in G_{q}(V)$, define $Z_{b}=\bigcap\{$ Zero $(\sigma) \mid \sigma \in E(b)\}$. Let $d(q, n)=\operatorname{dim} G_{q}(V)$. Let $\omega_{q, 1}$ be a normalized Kähler form on $G_{q}(V)$ such that, setting $\omega_{[q]}=\omega_{q, 1}^{d(q, n)}, \int_{G_{q}(V)} \omega_{[q]}=1$. The classifying map $c_{V}: Y \rightarrow G_{n-p}(V)$ is given by

$$
E\left(c_{V}(y)\right)=\{\sigma \in V \mid \sigma(y)=0\} \quad(y \in Y) .
$$

It can be easily shown that $Z_{b}=c_{V}^{-1}\left(S_{b}\right)$ for every $S_{b} \in \mathfrak{A}_{n-p, q}$. Here $S_{b}$ has codimension $s=p(q+1)$ in $G_{n-p}(V)$ (see [27]). Define

$$
\Omega_{p, q}=c_{V}^{*} \Psi\left(\omega_{[q]}\right) \in A_{\infty}^{s, s}(Y) .
$$

Then $\Omega_{p, q} \gg 0$ and $d \Omega_{p, q}=0$. 
Corollary 4.8. Assume $X$ satisfies (I) and $\varphi: X \rightarrow \boldsymbol{R}_{-\infty}$ is a $g$ concave exhaustion function. Let $W, V, q$ be as above, and $f: X \rightarrow Y$ be a meromorphic map. Assume for every branch $X_{j}$ of $X$ there is $a$ point $\left(x_{j}, b_{j}\right) \in\left(X_{j}-I_{f}\right) \times G_{q}(V)$ such that $\operatorname{codim}_{x_{j}} f_{0}^{-1}\left(Z_{b_{j}}\right)=s$. Assume $\chi \in A_{2}^{m-s, m-s}(X)$ is closed, nonnegative, and $\chi>0$ at some point of $X$, (if $m=s$, take $\chi=1$ ). Then for almost all $b \in G_{q}(V)$, $f_{0}^{-1}\left(Z_{b}\right)$ has pure codimension $s$ and

$$
\lim _{r \rightarrow \infty}^{\prime} \frac{N_{f}^{u}\left(r, r_{0}, Z_{b}\right)}{T_{f}^{u}\left(r, r_{0}, \Omega_{p, q}\right)}=1
$$

(Cf. the definitions in Theorem 4.4.)

Proof. The meromorphic map $c_{V} \circ f: X \rightarrow G_{n-p}(V)$ is almost adapted to $\mathfrak{A}_{n-p, q}$ by Corollary 1.4. Hence Corollary 4.7 yields the result.

TheOREM 4.9. Assume (I)-(III) and (IV-b). Assume $\varphi$ is a c.gconvex exhaustion function of $X$ such that (VI) holds with $\chi=\chi_{q}$ and $\Omega=\Omega_{s}$. (1) Assume for some positive form $\zeta \in A_{0}^{k-1, k-1}(N)$, one of the following conditions holds (over a $\phi$-admissible sequence $\sigma$ ):

(a) $A_{f, s-1}^{u}\left(r, \xi_{Y}\right)=o^{\prime}\left(T_{f, s}^{u}\left(r, 1, \Omega_{s}\right)\right)$

(b) $D_{f, s-1}^{u}\left(r, \xi_{Y}\right)=o^{\prime}\left(\int_{1}^{r} D_{f, s}^{u}\left(t, \Omega_{s}\right) u(t) d t+A_{f, s}^{u}\left(0, \Omega_{s}\right)\|u\|_{1}^{r}\right)$.

Then there is a set $N_{o} \subset N$ of measure zero such that for every $b \in$ $N-N_{o}$,

$$
\lim _{r \rightarrow \infty}^{\prime} \frac{N_{f, s}^{u}\left(r, r_{0}, S_{b}\right)}{T_{f, s}^{u}\left(r, r_{0}, \Omega_{s}\right)}=1 .
$$

(Here $r_{0}>0$ is an arbitrary constant.) (2) If $\varphi$ is g-semiparabolic and if $s=1$, the above conclusion holds for every $\phi$-admissible sequence $\sigma$.

Proof. For fixed $r^{\prime}>0, T_{f, s}^{u}\left(r, r^{\prime}, \Omega_{s}\right) \rightarrow \infty$ by (4.7), Lemmas 2.4 and 4.3. Let $\xi^{\prime} \in A_{\infty}^{k-1, k-1}(N)$ be a $d^{c} d$-closed positive form ([8, p. 961]). Then (4.6) and Lemma 2.2 imply

$$
D_{f, s-1}^{u}\left(r, \xi_{Y}^{\prime}\right) \leqq \text { const. } A_{f, s-1}^{u}\left(r, \xi_{Y}\right) \text {. }
$$

Therefore $(a) \Rightarrow(b) . \quad($ Similarly $(b) \Rightarrow(a)$.$) \quad Now (4.5) and Theorem$ 4.5 yield (4.15). Clearly (2) is a consequence of (1).

Corollary 4.10. Assume (I)-(III), (IV-b) and $\mathfrak{A}$ is strictly adm. of codimension 1. Assume $\varphi$ is an exhaustion function of $X$ such that one of the following holds:

(a) $\phi$ is c.g-convex and 


$$
A_{0}^{u}(r)=o^{\prime}\left(T_{f, 1}^{u}\left(r, 1, \Omega_{1}\right)\right) .
$$

(b) $\varphi$ is g-quasiparabolic.

If $m>1$, assume $\chi_{m} \not \equiv 0$. Assume either $Y$ is compact, or $d \omega_{N, 1}=0$ and $Y$ has a finite number of connectivity components. Then (4.15) holds for all $S_{b}$ with $b \in N-N_{\sigma}$. (Here $N_{\sigma}$ has measure zero, and in the case of (b), $\sigma$ is an arbitrary $\varphi$-admissible sequence.)

Proof. Since $\chi_{1} \geqq 0, \chi_{m} \not \equiv 0$ implies $\chi_{1}>0$ at some point of $X_{\text {reg }}$. Also, by hypotheses, $\Omega_{0}$ is a bounded function on $Y$. Hence the corollary follows from Theorem 4.9 and Lemma 4.3.

Lemma 4.11. Let $V, W: \boldsymbol{R}\left[c_{0}, \infty\right) \rightarrow \boldsymbol{R}[0, \infty)$, where $W$ is increasing $\neq 0$, and $V$ is measurable, locally bounded. Let $u, \gamma: \boldsymbol{R}\left[c_{0}, \infty\right) \rightarrow \boldsymbol{R}(0, \infty)$ be continuous functions with $\|\gamma u\|_{c_{0}}^{r} \rightarrow \infty$. Assume for some constants $\alpha>1, B \geqq 0$,

$$
\gamma(r)|V(r)-B|^{\alpha}=O(W(r)) \quad(r \longrightarrow \infty) .
$$

Let $E \subseteq \boldsymbol{R}\left[c_{0}, \infty\right)$ be a set of measure zero. Then there exists a sequence $\left\{r_{j}\right\}$ in $\boldsymbol{R}\left[c_{0}, \infty\right)-E$ tending to infinity such that

$$
V\left(r_{j}\right)=O\left(\|u W\|_{c_{0}}^{r_{j}}\right) \quad(j \longrightarrow \infty) .
$$

Proof. Put $H(r)=\|\gamma u\|_{c_{0}}^{r}$ and let $J$ be the inverse function of $H$ on $\boldsymbol{R}[0, \infty)$. There exist constants $K>0, r_{1} \geqq c_{0}$ such that

$$
\gamma(r)|V(r)-B|^{\alpha} \leqq K W(r) \quad\left(r \geqq r_{1}\right) .
$$

For $a>a_{1}=H\left(r_{1}\right)$ define $Q(a)=|V(J(a))-B|^{\alpha}$, and $P(a)=\|Q\|_{a_{1}}^{a}$. The case $P(a) \equiv 0$ is trivial, hence assume $P(a)>0$ for $a>a_{1}$. Then with $a=H(r)>a_{1}$,

$$
|V(r)-B| \leqq K\left[\left(P^{-\alpha} Q\right)(a)\right]^{1 / \alpha}\|u W\|_{r_{1}}^{r} .
$$

Since $P^{-\alpha} Q \in L^{1}\left(\left[a^{\prime}, \infty\right)\right)$ for large $a^{\prime}$, there exists a sequence $\left\{r_{j}\right\}$ in $\boldsymbol{R}\left[c_{0}, \infty\right)-E$ tending to infinity such that $\left(P^{-\alpha} Q\right)\left(H\left(r_{j}\right)\right)<2^{-j}$ for every $j$. From this the conclusion follows.

Corollary 4.12. Assume (I)-(III) and (IV-b). Assume $\varphi$ is a c.g-convex exhaustion function of $X$ such that (VI) holds with $\chi=\chi_{q}$ and $\Omega=\Omega_{s}$. Assume there exists a positive form $\xi \in A_{0}^{k-1, k-1}(N)$ and a positive continuous $\gamma: \boldsymbol{R}\left[a_{0}, \infty\right) \rightarrow \boldsymbol{R}$ with $\|\gamma u\|_{a_{0}}^{r} \rightarrow \infty$ such that for some constants $\alpha>1, B \geqq 0$, one of the following holds:

(a) $\gamma(r)\left|D_{f, s-1}^{u}\left(r, \xi_{Y}\right)-B\right|^{\alpha}=O\left(D_{f, s}^{u}\left(r, \Omega_{s}\right)\right)$.

(b) $\gamma(r)\left|A_{f, s-1}^{u}\left(r, \xi_{Y}\right)-B\right|^{\alpha}=O\left(A_{f, s}^{u}\left(r, \Omega_{s}\right)\right)$.

Then there exists a $\varphi$-admissible sequence $\sigma$ for which (4.15) holds for almost every $S_{b} \in \mathfrak{A}$. 
Proof. Apply Lemma 4.11 and Theorem 4.9.

To give some applications of the preceding results, consider the following:

1. Let $f: X \rightarrow G_{p}(V)$ be a meromorphic map (where $V$ has $\operatorname{dim} n+1$ ) and $\mathfrak{A}=\mathscr{D}_{p, n}$. If $n=1$, assume $f$ is nondegenerate; if $n>1$, assume every branch of $X$ contains a point $x \notin I_{f}$ for which there is a $\Sigma_{b} \in$ $\mathscr{D}_{p, n}$ with $\operatorname{dim}_{x} f_{0}^{-1}\left(\Sigma_{b}\right)=m-1$. Assume $\varphi: X \rightarrow R$ is an exhaustion function such that either 4.10 (a) or 4.10 (b) (with $\Omega_{1}=\Psi\left(\omega_{[n-p-1]}\right)$ ) holds. If $m>1$, assume $\chi_{m} \neq \equiv$. Then (4.15) holds for almost every $\Sigma_{b} \in \mathscr{D}_{p, n}$.

2. Theorem 4.6 of Stoll [26] for a family $\left\{S_{v}\right\}_{v \in E(A)}$ of Schubert zeros holds for a c.g-convex space $(X, \varphi)$ (under conditions similar to (4.3), ibid.) in the stronger sense that the valence of almost all $S_{v}$ grows at the same rate as the characteristics of $f$. Especially, the theorem holds if $\varphi$ is $g$-quasiparabolic and $q$ (see [26, assump. $(13)])=m-1$. This can be proved using Theorem 4.4 and Lemma 3.2 .

3. (Cf. Stoll $[23,9.5]$.) Assume (I)-(IV-a) with $X$ nonsingular, connected, and $\mathfrak{A}$ strictly admissible of codimension 1 . Assume $\chi \in$ $A_{\infty}^{m-1, m-1}(X)$ is closed and positive. Let $\sigma=\left\{G_{j}\right\}_{j=0}^{\infty}$ be a sequence of domains in $X$ such that $\varnothing \neq G_{j} \subset G_{j+1}, X-G_{0}$ has no compact component, $d G_{j}=\partial G_{j}$, and $\cup G_{j}=X$. Then there exist functions $\psi_{j}: X \rightarrow$ $\boldsymbol{R}(j \geqq 1)$ solving the Dirichlet problem

$$
\chi \wedge d d^{c} \psi_{j}=0 \quad \text { on } \quad G_{j}-\bar{G}_{0},
$$

with $\psi_{j}\left|\dot{G}_{0}=1, \psi_{j}\right| X-G_{j}=0$. The capacity of $G_{j}$ (relative to $\chi$ ) is defined by

$$
C\left(G_{j}\right)=\int_{G_{j}-\bar{G}_{0}} \chi \wedge d \psi_{j} \wedge d^{c} \psi_{j} \quad(j \geqq 1) .
$$

It follows that $\psi_{j} \leqq \psi_{j+1}$, and $0<C\left(G_{j+1}\right) \leqq C\left(G_{j}\right)([23,9.3])$. Assume either (a) $C\left(G_{j}\right) \rightarrow 0$ or (b) $T_{f}\left(G_{j}\right)=\int_{G_{j}} \psi_{j} \chi \wedge f^{*} \Omega \rightarrow \infty$. Then for almost every $S_{b} \in \mathfrak{A}$,

$$
\lim _{j \rightarrow \infty} \frac{N_{f}\left(G_{j}, S_{b}, \psi_{j} \chi\right)}{T_{f}\left(G_{j}\right)}=1
$$

This follows from (3.3), Lemma 2.4 and the proof of Theorem 4.4, observing that for $j \geqq 1$,

$$
\int_{d G_{0}} f^{*} A_{Y} \wedge d^{\perp} \psi_{j} \wedge \chi \leqq \text { const. } C\left(G_{j}\right)
$$




\section{REFERENCES}

1. A. Andreotti and W. Stoll, Analytic and algebraic dependence of meromorphic functions, Lecture Notes in Mathematics 234. Springer-Verlag, Berlin and New York, 1971.

2. S. Bochner and W. T. Martin, Several Complex Variables, Princeton Mathematical Series, Vol. 10, Princeton, 1948.

3. R. Bott and S. S. Chern, Hermitian vector bundles and the equidistribution of the zeros of their holomorphic sections, Acta Math., 114 (1965), 71-112.

4. S. S. Chern, The integrated form of the first main theorem for complex analytic mappings in several variables, Ann. of Math., (2) 71 (1960), 536-551.

5. - Complex manifolds without potential theory, Van Nostrand Math. Studies, no. 15, Van Nostrand, Princeton, N. J., 1967.

6. - Holomorphic curves and minimal surfaces, Carolina Conference Proc., Holomorphic Mappings and Minimal Surfaces, Chapel Hill, N. C., 1970, 1-28.

7. M. Cowen, Hermitian vector bundles and value distribution for Schubert cycles, Trans. Amer. Math. Soc., 180 (1973), 189-228.

8. I. M. Dektyarev, The general first fundamental theorem of value distribution theory, Dokl, Adak. Nauk SSSR, 193 (1970) (Soviet Math. Dokl. 11 (1970), 961-963).

9. —_ Problems of value distribution in dimensions higher than unity, Uspekhi Matem, Nauk 25, no. 6, 53-84 (1970) (Russian Math. Survey, Vol. 25, no. 6 (1970), 51-82).

10. P. Griffiths, Two theorems on extensions of holomorphic mappings, Invent. Math., 14 (1971), 27-62.

11. - Holomorphic mappings: Survey of some results and discussion on open problems, Bull. Amer. Math. Soc., 78 (1972), 374-382.

12. P. Griffiths and J. King, Nevanlinna theory and holomorphic mappings between algebraic varieties, Acta Math., 130 (1973), 145-220.

13. J. Hirschfelder, The first main theorem of value distribution in several variables, Invent. Math., 8 (1969), 1-33.

14. On Wu's form of the first main theorem of value distribution, Proc. Amer. Math. Soc., 23 (1969), 548-554.

15. L. Hormander, Linear partial differential operators, Die Grundlehren der mathematischen Wissenschaften, Band 116, Springer-Verlag, Berlin-Gottingen-Heidelberg, 1963. 16. Y. Matsushima, On a problem of Stoll concerning a cohomology map from a flag manifold into a Grasmann manifold, Osaka J. Math., 13 (1976), 231-269.

17. Y. Matsushima and W. Stoll, Ample vector bundles on compact complex spaces, Rice University Studies, 59 (1973), 71-107.

18. R. Remmert, Holomorphe und meromorphe Abbildungen komplexer Räume, Math. Ann., 33 (1957), 328-370.

19. M. Schechter, Differentiability of solutions of elliptic problems with respect to parameters, (preprint).

20. K. Stein, Maximale holomorphe und meromorphe Abbildungen, II, Amer. J. Math., 86 (1964), 823-868.

21. W. Stoll, A general first main theorem of value distribution, Acta Math., 118 (1967), 111-191.

22. - About value distribution of holomorphic maps into projective space, Acta Math., 123 (1969), 83-114.

23. - Value distribution of holomorphic maps into compact complex manifolds, Lecture Notes in Mathematics 135. Springer-Verlag, Berlin-Heidelberg-New York, 1970. 24. - Deficit and Bezout estimates, Value Distribution Theorem Part B, Pure and Appl. Math., 25, Marcel Dekker, New York 1973.

25. - Value distribution theory on parabolic spaces, Lecture Notes in Mathematics

600. Springer-Verlag, Berlin-Heidelberg-New York, 1977. 
26. W. Stoll, A Casorati Weierstrass theorem for Schubert zeros of semi-ample holomorphic vector bundles, Memoire dell' Accademia Nazionale dei Lincei (to appear).

27. - Invariant forms on Grassmann manifolds, Annals of Math. Studies, 89, pp. 113, Princeton University Press, Princeton, NJ 1977.

28. C. Tung, The first main theorem of value distribution on complex spaces, Memoire dell' Accademia Nazionale doi Lincei (to appear).

29. - On the equidistribution theory of holomorphic maps, Proc. Symp. Pure Math., 30 (1976), 265-269; Amer. Math. Soc., Providence, R. I.

30. - A generalized Gauss-Bonnet formula for Schubert type analysic sets, (preprint).

31. $\mathrm{H}$. Wu, Remarks on the first main theorem of equidistribution theory, I-IV, J. Diff. Geometry 2 (1968), 197-202, 369-384; ibid. 3 (1969), 83-94, 433-446.

Received November 15, 1977. Partially sapported by NSF Grant MCS 76-08478.

COLUMBIA UNIVERSITY

NEW YoRK, NY 10027

Current address: Department of Mathematics

SUNY

Buffalo, NY 14214 


\title{
PACIFIC JOURNAL OF MATHEMATICS
}

\section{EDITORS}

RICHARD ARENS (Managing Editor)

University of California

Los Angeles, CA 90024

Charles W. Curtis

University of Oregon

Eugene, OR 97403

C. C. MOORE

University of California

Berkeley, CA 94720

\section{J. DUGUNDJI}

Department of Mathematics University of Southern California Los Angeles, CA 90007

R. Finn and J. Milgram

Stanford University

Stanford, CA 94305

\section{ASSOCIATE EDITORS}
E. F. BECKENBACH
B. H. NeumanN
F. WOLF
K. YOSHIDA

\section{SUPPORTING INSTITUTIONS}

\author{
UNIVERSITY OF BRITISH COLUMBIA \\ CALIFORNIA INSTITUTE OF TECHNOLOGY \\ UNIVERSITY OF CALIFORNIA \\ MONTANA STATE UNIVERSITY \\ UNIVERSITY OF NEVADA, RENO \\ NEW MEXICO STATE UNIVERSITY \\ OREGON STATE UNIVERSITY \\ UNIVERSITY OF OREGON
}

\author{
UNIVERSITY OF SOUTHERN CALIFORNIA \\ STANFORD UNIVERSITY \\ UNIVERSITY OF HAWAII \\ UNIVERSITY OF TOKYO \\ UNIVERSITY OF UTAH \\ WASHINGTON STATE UNIVERSITY \\ UNIVERSITY OF WASHINGTON
}

The Supporting Institutions listed above contribute to the cost of publication of this Journal, but they are not owners or publishers and have no responsibility for its content or policies.

Mathematical papers intended for publication in the Pacific Journal of Mathematics should be in typed form or offset-reproduced, (not dittoed), double spaced with large margins. Please do not use built up fractions in the text of the manuscript. However, you may use them in the displayed equations. Underline Greek letters in red, German in green, and script in blue. The first paragraph or two must be capable of being used separately as a synopsis of the entire paper. Items of the bibliography should not be cited there unless absolutely necessary, in which case they must be identified by author and journal, rather than by item number. Manuscripts, in triplicate, may be sent to any one of the editors. Please classify according to the scheme of Math. Reviews, Index to Vol. 39. All other communications should be addressed to the managing editor, or Elaine Barth, University of California, Los Angeles, California, 90024.

50 reprints to each author are provided free for each article, only if page charges have been substantially paid. Additional copies may be obtained at cost in multiples of 50 .

The Pacific Journal of Mathematics is issued monthly as of January 1966. Regular subscription rate: $\$ 72.00$ a year (6 Vols., 12 issues). Special rate: $\$ 36.00$ a year to individual members of supporting institutions.

Subscriptions, orders for numbers issued in the last three calendar years, and changes of address should be sent to Pacific Journal of Mathematics, P.O. Box 969, Carmel Valley, CA 93924, U.S.A. Older back numbers obtainable from Kraus Periodicals Co., Route 100, Millwood, NY 10546.

PUBLISHED BY PACIFIC JOURNAL OF MATHEMATICS, A NON-PROFIT CORPORATION

Printed at Kokusai Bunken Insatsusha (International Academic Printing Co., Ltd.). 8-8, 3-chome, Takadanobaba, Shinjuku-ku, Tokyo 160, Japan.

Copyright (C) 1978 by Pacific Journal of Mathematics Manufactured and first issued in Japan 


\section{Pacific Journal of Mathematics \\ Vol. 78, No. $2 \quad$ April, 1978}

Su-Shing Chen, Weak rigidity of compact negatively curved manifolds .... 273

Heinz Otto Cordes and D. A. Williams, An algebra of pseudodifferential operators with nonsmooth symbol ....................... 279

Herbert Paul Halpern, Normal expectations and integral decomposition of type III von Neumann algebras ......................... 291

G. Hochschild, On representing analytic groups with their

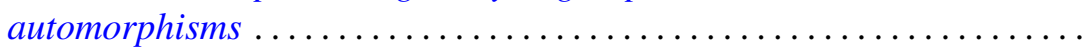

Dean G. Hoffman and David Anthony Klarner, Sets of integers closed under affine operators - the closure of finite sets ....................

Simeon Ivanov, On holomorphic relative inverses of operator-valued

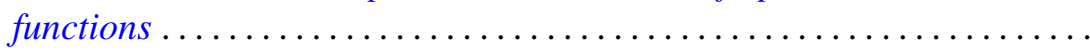

O. P. Juneja and M. L. Mogra, Radii of convexity for certain classes of

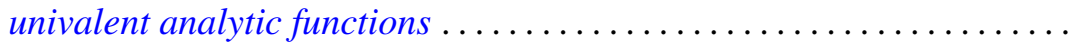

Hadi Kharaghani, The evolution of bounded linear functionals with application to invariant means.......................... 369

Jack W. Macki, A singular nonlinear boundary value problem .......... 375

A. W. Mason and Walter Wilson Stothers, Remarks on a theorem of $L$. Greenberg on the modular group ........................ 385

Kevin Mor McCrimmon, Peirce ideals in Jordan algebras . . . . . . . . . . . 397

John C. Morgan, II, On the absolute Baire property ................ 415

Gerard J. Murphy, Commutative non-Archimedean $C^{*}$-algebras ...

Masafumi Okumura, Submanifolds with L-flat normal connection of the

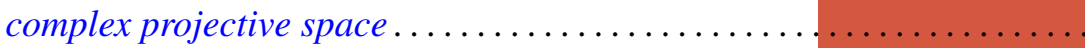

Chull Park and David Lee Skoug, Distribution estimates of barrier-crossing probabilities of the Yeh-Wiener process ...............

Irving Reiner, Invariants of integral representations ........

Phillip Schultz, The typeset and cotypeset of a rank 2 abelian group ..... 503

John Brendan Sullivan, Representations of Witt groups ....

Chia-Chi Tung, Equidistribution theory in higher dimensions . . . 